Stanicki Pawel, Szarpak Julita, Wieteska Małgorzata, Kaczyńska Agnieszka, Piecewicz-Szczęsna Halina. Specifics of COVID-19 in pregnant women and their children - a review. Journal of Education, Health and Sport. 2020;10(9):103-110. eISSN 2391-8306. DOI http://dx.doi.org/10.12775/JEHS.2020.10.09.011

https://apcz.umk.pl/czasopisma/index.php/JEHS/article/view/JEHS.2020.10.09.011

https://zenodo.org/record/4014369

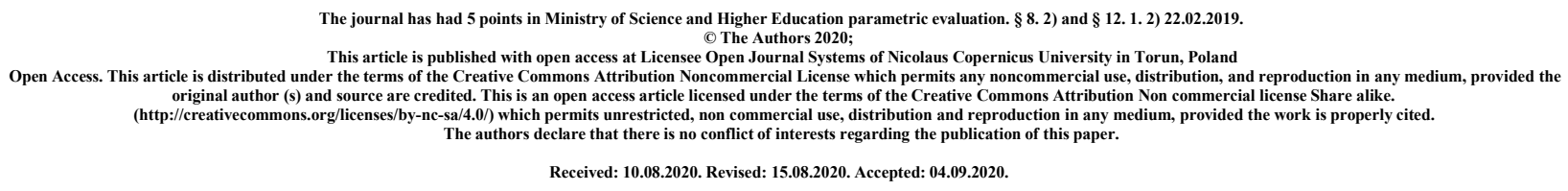

\title{
Specifics of COVID-19 in pregnant women and their children - a review
}

\author{
Pawel Stanicki', Julita Szarpak ${ }^{1}$, Małgorzata Wieteska', Agnieszka Kaczyńska ${ }^{1}$, \\ Halina Piecewicz-Szczęsna²
}

1. Student Research Circle at the Department of Epidemiology and Clinical Research Methodology, Medical University of Lublin, Poland

2. Chair and Department of Epidemiology and Clinical Methodology, Medical University of Lublin, Poland

ORCID ID and E-mail:

Paweł Stanicki https://orcid.org/0000-0002-4942-9030; stanicki99@gmail.com Julita Szarpak https://orcid.org/0000-0002-5091-0235; julita.szarpak@gmail.com Małgorzata Wieteska https://orcid.org/0000-0002-1566-7559; malgosia.wieteska@onet.eu Agnieszka Kaczyńska https://orcid.org/0000-0002-8943-9401; agaagnieszkakk@gmail.com Halina Piecewicz-Szczęsna https://orcid.org/0000-0002-0573-7226; halina.piecewiczszczesna@umlub.pl

\footnotetext{
Abstract:

Introduction and purpose: The first cases of SARS-CoV-2 infection were recorded in the last months of 2019 in the Chinese city Wuhan. Since then, COVID-19 has spread throughout the world. Still little is known about the perinatal aspects of COVID-19. Objective of this article is to present the course of coronavirus disease in pregnant women with particular emphasis on the occurrence of intrauterine infection and preterm births.
} 
Brief description of the state of knowledge: SARS-CoV-2 calling COVID-19 probably comes from bats sold at the Wuhan market. This virus is highly contagious, it spreads from person to person. The most common symptoms of infection include: fever, cough and ground-glass opacity (GGO) in chest radiographs. The analyzes carried out so far show that the course of the disease in pregnant women is similar to that of non-pregnant patients. Women present typical signs of infection. Most researchers also exclude the presence of intrauterine infection, although there have been reports of isolated cases. The percentage of premature births in women with COVID-19 is higher than average.

Summary: The course of coronavirus disease in pregnant women is similar to that of other infected women. While most studies do exclude the presence of a vertical infection, there are reports of severe course of the disease and possible vertical infection. Patients with COVID-19 may be more prone to premature delivery, however, to confirm this thesis, it is necessary to examine a larger number of pregnant women.

Key words: pregnancy; SARS virus; vertical transmission

\section{Introduction and purpose}

At the end of 2019, the first cases of infection with the previously unknown SARS-CoV-2 (severe acute respiratory syndrome coronavirus 2) virus were recorded in the Chinese city of Wuhan. This pathogen causes an acute inflammatory respiratory disease known as COVID-19 (coronavirus disease 2019). There have been epidemics in the past caused by SARS-CoV and MERS-CoV (Middle East respiratory syndrome) coronaviruses. These two were together responsible for over 10,000 cases in the last 20 years, with a mortality rate of $10 \%$ at SARS-CoV and $37 \%$ at MERS-CoV, respectively [1]. This number appears to be small compared to 10 million confirmed SARS-CoV-2 infections and over 500.000 deaths from COVID-19 within approximately 7.5 months of the outbreak [2].

An estimated $2 \%$ of admitted patients are pregnant women (data analysis of 21.9 thousand admissions in New York for March 2020) [3]. Research over the years has allowed better identification of threats to women infected with both SARS-CoV [4-7] and (to a lesser extent) MERS-CoV [8,9]. It is different in the case of SARS-CoV-2. This virus has been discovered recently and we have limited data on the risks to women associated with it.

Following review covers the subject of SARS-CoV-2 infection. It aims to present the latest research results on the course of pregnancy and childbirth in women with COVID-19. The most important work points are the assessment of the occurrence of intrauterine infection and the risk of preterm birth.

\section{Description of the state of knowledge \\ General characteristics of COVID-19}

COVID-19 disease is caused by the SARS-CoV-2 virus. The pathogen probably originated from bats sold at a market in Wuhan. The incubation period lasts from 1 to 14 days (usually from 3 to 7). The virus is very contagious. It transmits human-to-human by direct contact and droplets [10]. After entering the body, it binds to angiotensin-converting enzyme 2 (ACE2), which is also a SARS-CoV binding site [11]. Viruses have identical structure in nearly $80 \%$ [12]. 
After entering the cell, viral RNA is released into the cytoplasm and amplified by RTC (replicationtranscription complex) and then, after the submission of new copies of the pathogen, released by fusion with the cell membrane. This leads to the activation of the immune response. Excessive activation of macrophages, monocytes and cytokines produced in large amounts may lead to the development of acute respiratory distress syndrome (ARDS) [13].

The most common symptoms of COVID-19 are fever, runny nose and coughing [10]. In severe cases, pneumonia, ARDS, and renal failure occur [13]. Elderly patients with comorbid diseases such as hypertension, diabetes mellitus, and cardiovascular diseases are much more exposed to the acute course of COVID-19. A worse development of the disease is also observed in the presence of immunodeficiencies and chronic respiratory diseases (chronic obstructive pulmonary disease, cystic fibrosis) [10]. Research indicates that older people are more susceptible to COVID-19 - the median age of patients is estimated at 47-59 years [11]. COVID-19 has a mortality rate of approx. $4.3 \%$ [14], which is significantly lower than in the case of SARS (9.6\%) and MERS (approx. 35\%) [11].

\section{Review of studies in pregnant patients affected by COVID-19}

A number of studies and retrospective analysis have been performed in pregnant women infected with SARS-CoV-2. They took into account the occurrence of both maternal and fetal complications.

Liu D. et al. analyzed the results of computed tomography (CT) of 15 pregnant women admitted with diagnosed coronavirus pneumonia at the turn of January and February 2020 [15]. 11 patients were born during the study period - 10 by caesarean section and 1 by vaginal delivery. The most common symptoms of pneumonia were fever and cough. There were no serious perinatal complications. Among the changes in CT, main observation were areas of ground-glass opacity (GGO). They may indicate a partial filling of air spaces in the lungs by exudative fluid, as well as interstitial thickening of alveoli [16]. Comparative analysis of CT images taken before and after delivery did not show any signs of exacerbation of pneumonia after childbirth.

Another study in Italy conducted a retrospective analysis of medical records of 42 pregnant women [17]. The most commonly reported symptom was fever. Pneumonia was found in 19 of them, of which 7 required oxygen support and 4 were admitted to the intensive care unit (ICU). 24 women delivered vaginally and 18 had a cesarean section. Eleven children were born before the 37th week of pregnancy, of which two obtained an Apgar score lower than 7 in the fifth minute of life. Two women who were diagnosed with COVID-19 after delivery, breastfed their children without wearing a surgical mask, which was recommended by physicians. Samples collected from their newborns were found to be positive for SARS-CoV-2. One of them developed gastrointestinal and then respiratory symptoms, which resolved after one day of mechanical ventilation. Samples taken from him in the first hours of life were ambiguous and tests carried out 3 days later gave a positive result. Except for these three cases, no neonatal SARS-CoV-2 infection was reported.

Also, an analysis conducted by Chen et al. indicates the absence of vertical transmission of the SARS-CoV-2 virus [18]. Researchers reviewed retrospectively the medical records of nine women admitted to Zhongnan Hospital who found coronavirus with qRT-PCR (quantitative reverse transcription polymerase chain reaction). All women were in the third trimester of pregnancy and their age ranged from 26 to 40 years. One patient developed gestational hypertension at 27 gestational weeks and another developed pre-eclampsia at week 31. 
In addition, one of the women had was infected with influenza virus upon admission to the hospital. The patients presented various symptoms of upper respiratory tract infection: fever $(n=7$, in which none of the women exceeded $\left.39^{\circ} \mathrm{C}\right)$, cough $(n=4)$, myalgia $(n=3)$ and sore throat $(n=2)$. However, none of them developed severe pneumonia. Similarly, neither of them needed mechanical ventilation. Laboratory studies have shown lymphopenia $(n=5)$ and elevated levels of C-reactive protein $(n=6)$, alanine (ALT) and aspartate aminotransferase (AST) $(n=3)$. CT scan showed the presence of multiple, non-uniform GGO shadows in the lungs of eight patients. All children were born alive (caesarean deliveries), reaching 8-10 points on the Apgar scale. Two women developed premature rupture of the membranes and the examination of amniotic fluid, umbilical cord blood, neonatal throat swab and milk of six patients did not show SARS-CoV-2 in any of the samples.

Khan et al. Examined 17 pregnant women admitted to the hospital in Hubei between January 25 and February 15, 2020. COVID-19 was found in each of them by qRT-PCR or / and CT. They were treated with antibiotics $(n=17)$, hormonal $(n=8)$ and antiviral $(n=15)$ drugs. All patients gave birth via caesarean section. Symptoms that most often occurred in mothers were: cough $(n=6)$, fever $(n=5)$ and diarrhea $(n=3)$. No serious perinatal complications were observed, children on the Apgar scale scored 9 and 10. After birth, a throat swab was taken from them. In 15 cases no COVID-19 infection was found, the other two were suspected but no unequivocally positive result was obtained. Five newborns were diagnosed with pneumonia, and three of them were born prematurely [19].

A retrospective study conducted by $\mathrm{Yu}$ et al. included 7 patients admitted to the Tongji Hospital (Wuhan) between 01/01/2020 and 8/02/2020 [20]. All of them were found to have SARSCoV-2 virus due to rt-PCR examination of throat swabs. The age of the patients ranged from 29 to 34 years. During admission, they presented fever $(n=6)$, cough $(n=1)$, shortness of breath $(n=1)$ and diarrhea $(\mathrm{n}=1)$. Two women were chronically ill with hypothyroidism and polycystic ovary syndrome, respectively. Two patients were also co-infected with H1N1 virus and one patient with Legionella pneumophila. In addition, three pregnant women had uterine scarring. Laboratory tests showed elevated levels of neutrophils $(n=5)$, D-dimers $(n=7)$, AST and / or ALT $(n=2)$ and protein $C(n=7)$ while lymphocytes $(n=5)$ and platelets $(n=2)$ were reduced. All seven women had caesarean delivery, they were carried out without any serious complications (birth weight and Apgar scores normal). Four of the newborns left the hospital and were not tested for the presence of SARS-CoV-2, however they did not show fever, pathological jaundice or other symptoms that could indicate infection. The throat swabs of the other three were tested for the presence of the virus. In two of them the result was negative, while in one, after 36 hours from delivery, it was positive. Tests were carried out for the presence of viral nucleic acid in the placenta and umbilical cord blood of this patient, but the results were negative. This suggests that he became ill after birth, and intrauterine infection did not occur. The newborn was transported to a children's hospital, where he was diagnosed with mild shortness of breath and slight changes in lung x-ray radiography. These symptoms resolved quickly - he was discharged after two weeks when he twice obtained a negative test for nucleic acid of the virus.

\section{Severe cases of COVID-19 in pregnant women}

While most pregnant women appear to be undergoing mild coronavirus infection, there have also been reports of severe COVID-19 cases. A description of such case appears in a paper describing 7 pregnant women presenting to hospital in New York City [21]. Of the 7 patients, 4 were admitted to the hospital, including 2 who required ICU care. 
One of them (at 37 weeks' gestation) was adopted with the intention of inducing pregnancy due to poorly controlled type 2 diabetes and intrahepatic cholestasis of pregnancy. During delivery, the patient's temperature increased to $38.5^{\circ} \mathrm{C}$. She underwent a caesarean section, during which an atony of the uterus occurred. 1.5 liters of blood was lost and the patient was intubated. After admission to ICU, she was treated with hydorksychloroquine and her condition was systematically improving - she was discharged on day 4 after delivery. Her child tested negative for SARS-CoV-2 on day 1 of life. The second woman, also at 37 weeks' gestation, was admitted due to worsening of chronic hypertension. About 25 hours after cesarean delivery, she developed a cough that progressed to respiratory failure. Additionally, her body temperature increased $\left(39.4^{\circ} \mathrm{C}\right)$ and pulmonary edema appeared. Severe hypertension $(200 / 90 \mathrm{~mm} \mathrm{Hg})$ occurred, which resulted in the patient being transferred to the ICU. Like the first woman, this one was also treated with hydroxychloroquine and antibiotics, and her child SARS-CoV-2 test came out negative.

The infection in a pregnant woman described by Alzamora et al [22] had a similar course. The patient with COVID-19 admitted to the hospital was in her third pregnancy. She had diabetes and her BMI was $35 \mathrm{~kg} / \mathrm{m}^{2}$. She complained of fatigue and fever, was intubated and mechanically ventilated as a result of developing dyspnea. The caesarean section was performed and child obtained 6 and 8 points on the Apgar scale - in 1 and 5 minutes respectively. The boy was also intubated and isolated before contacting his mother. The test result of the rt-PCR for SARS-CoV-2 in a nasopharyngeal swab of a newborn taken 16 hours after delivery came out positive.

Serious complications in the newborn have also been described by Zhu et al. [23]. This study took into account the results of 10 newborns and their mothers - patients with confirmed SARS-CoV-2 infection. The women were examined before treatment - chest CT revealed typical lesions characteristic for a viral airway inflammation: blurred lung borders and bilateral GGO. Seven women had a cesarean section and two delivered vaginally. Complications that occurred at delivery were: premature rupture of the membranes $(n=3)$, abnormal amniotic fluid $(n=2)$, umbilical cord $(n=2)$ and placenta pathologies $(n=1)$. Six children were born prematurely. This is a relatively large percentage. The problem of prematurity affects 5 to $18 \%$ of newborns worldwide [24]. Given this review and the 10 studies it contains (Table 1), the percentage of children born before the 37 th week of content is $20,4 \%$. One premature child had shortness of breath 30 minutes after delivery. Eight days later, he developed shock, disseminated intravascular coagulation syndrome and multi-organ failure. Despite the administration of red blood cells, platelets and plasma, he died on the ninth day after delivery.

\section{Comparison of COVID-19 in pregnant and non-pregnant women}

The risk groups for COVID-19 include the elderly and the chronically ill [10]. To determine if pregnant women are more exposed to complications, comparative analysis are performed.

Qiancheng et al. Made a retrospective summary of the medical records of 82 women, including 28 pregnant and 54 in reproductive age, not pregnant [25]. The severity of pneumonia was comparable in both groups. With the exception for two patients (pregnant) with mild disease and three ( 2 pregnant, 1 non-pregnant) presenting severe form of inflammation, all women were classified as having moderate severity. In the group of pregnant women, leukocytosis and elevated $\mathrm{C}$ protein levels were observed more often. On the other hand, hemoglobin and albumin levels were lower than in the group of non-pregnant women. There were no differences in procalcitonin levels between groups. Chest CT scan revealed the presence of GGO in all women except two pregnant patients. 
During the review period, 22 women gave birth, 2 continued their pregnancy and 4 terminated it voluntarily. Of the 23 children born (one pair of twins), only one was born before 37 weeks of gestation. There were no serious perinatal complications. None of the children were found to have SARS-CoV-2 infection in rt-PCR. Researchers deny the occurrence of significant differences in the course of the disease in pregnant and non-pregnant women.

Another study comparing CT images of infected patients was conducted by a team led by Liu H. [26]. A retrospective review of the results of 59 patients with COVID-19 was performed, including 41 pregnant women (16 were diagnosed based on laboratory tests, 25 based on clinical symptoms), 11 non-pregnant adults and 4 children. The median age of pregnant women was 30 years (22-42). A higher incidence of elevated body temperature was observed in this group compared to non-pregnant adults $(\mathrm{p}<0.001)$. Similarly, leukocytosis and an increase in the percentage of neutrophils were detected in pregnant women more often than in the control group ( $p$ $<0.001$ ). Similarly to the study done by Liu D. [15], here also GGO type changes in CT of patients were observed. At the same time, consolidation (mixed GGO with consolidation) could be observed in pregnant women, while in the group of adult non-pregnant patients more GGO with reticulation appeared. In newborns, no virus infection or other abnormalities were observed after delivery [26].

Interesting results were also obtained by a group of scientists led by $\mathrm{Wu}$. They reviewed the medical documentation of eight pregnant women with COVID-19, additionally comparing them with the results of five pregnant women in which infection was excluded [27]. The study mainly focused on comparing COVID-19 symptoms in women before and after delivery. Seven of them did not present any typical abnormalities before birth. Three of them had a fever after delivery. Laboratory tests performed before delivery showed normal (or very close to normal) white blood cells and lymphocytes levels in all eight infected patients. After delivery, there was a drastic increase in the number of leukocytes (in six patients) and a decrease in the number of lymphocytes (in five patients). For comparison, a similar tendency on a much smaller scale or no change at all was observed in a group of five healthy women. Comparable, observation of protein $\mathrm{C}$ and D-dimer concentration behavior showed a significant increase after birth, but was more pronounced in patients with SARS-CoV-2. The results of this study suggest the possibility of increased susceptibility of pregnant women with COVID-19 to postpartum complications. The basis of this phenomenon can be seen in the mother's immune changes during pregnancy [28] and the impact of the virus on the immune system [13].

Tab. 1 Results of clinical trials in pregnant women

\begin{tabular}{|c|c|c|c|c|c|c|}
\hline Title & Authors & Time of studv / & Research group & Presence of & Premature birth & Other results \\
\hline Pregnancv and & Liu D. Li L. Wu & Januarv 20.2020 - & 15 pregnant & Not tested & Occurred in 3 out & GGO in the CT \\
\hline Vaginal delivery & Ferrazzi E, & March 1-20, 2020 & 42 pregnant & Not found in 39 of & Occurred in 11 of & - \\
\hline Clinical & Chen H, Guo J, & January 20-31, & 9 pregnant women & Not found & Occurred in 3 out & Complications \\
\hline Association of & Khan S. Jun L. & Januarv 25 - & 17 pregnant & Not found. two & Occurred in 3 out & Pneumonia \\
\hline Clinical features & Yu N, Li W, Kang & January 1 - & 7 pregnant women & Not found in 6 out & Not found & One infected \\
\hline Coronavirus & Liu Y, Chen H, & March 18-19. & 7 pregnant women & Not found & Not found & A severe case of \\
\hline Clinical analvsis & Zhu H. Wang L. & Januarv 20 - & 9 pregnant women & Not found & Occurred in 6 out & Serious \\
\hline Coronavirus & Qiancheng X, Jian & January 15 - & 82 women, & Not found & Occurred in 1 out & A comparative \\
\hline Clinical and CT & Liu H, Liu F, Li J, & January 27 - & 59 patients, & Not found & Not given & 14 of 16 patients \\
\hline Clinical & Wu C, Yang W. & 23 stycznia - 10 & 8 pregnant women & Not tested & Occurred in 2 out & Comparison of th \\
\hline
\end{tabular}




\section{Summary}

The epidemiological crisis caused by the SARS-CoV-2 virus is an extremely difficult challenge for modern medicine. Factors such as high infectivity and asymptomatic course in many cases also make it problematic to control the spread of the disease. This review aims to clarify the specifics of COVID-19 in pregnant women and their children. The studies collected here do not indicate the presence of intrauterine SARS-CoV-2 infection. Similarly, other reviews seemed to exclude this route of infection [29-31]. However, there are reports of infection in newborns. It cannot, therefore, unequivocally rule out intrauterine SARS-CoV-2 infection, especially considering the small number of pregnant women that have been screened so far.

The percentage of prematurity in those infected with SARS-CoV-2 is higher than the average among healthy women, however, the research sample is small in this case and further studies are needed to confirm the association of COVID-19 with an increased risk of premature delivery.

Studies suggest that the course of COVID-19 in most pregnant women is very similar to that of other patients [30]. Most of them undergo mild infection and the most common symptoms include fever, cough and GGO in computed tomography of the lungs. Nonetheless, reports of severe cases and serious perinatal complications suggest conducting further research to better understand the specificity of the disease and adapt appropriate treatment.

\section{List of references}

1. Huang C, Wang Y, Li X et al. Clinical features of patients infected with 2019 novel coronavirus in Wuhan, China. Lancet. 2020;395(10223):497-506. https://doi.org/10.1016/S0140-6736(20)30183-5

2. Coronavirus disease (COVID-19) Situation Report - 163, World Health Organization. https://www.who.int/docs/default-source/coronaviruse/situation-reports/20200701-covid-19-sitrep163.pdf?sfvrsn=c202f05b_ 2 (access: 1.07 .2020$)$

3. Tekbali A, Grünebaum A, Saraya A et al. Pregnant vs nonpregnant severe acute respiratory syndrome coronavirus 2 and coronavirus disease 2019 hospital admissions: the first 4 weeks in New York. [letter]. Am J Obstet Gynecol. 2020;223(1):126-127. https://doi.org/10.1016/j.ajog.2020.04.012

4. de Wit E, van Doremalen N, Falzarano D et al. SARS and MERS: recent insights into emerging coronaviruses. Nat Rev Microbiol. 2016;14(8):523-534. https://doi.org/10.1038/nrmicro.2016.81

5. Báez-Santos YM, St John SE, Mesecar AD. The SARS-coronavirus papain-like protease: structure, function and inhibition by designed antiviral compounds. Antiviral Res. 2015;11521-38.

https://doi.org/10.1016/j.antiviral.2014.12.015

6. Yuan Y, Cao D, Zhang Y et al. Cryo-EM structures of MERS-CoV and SARS-CoV spike glycoproteins reveal the dynamic receptor binding domains. Nat Commun. 2017;8:15092. https://doi.org/10.1038/ncomms15092

7. Millet JK, Whittaker GR. Physiological and molecular triggers for SARS-CoV membrane fusion and entry into host cells. Virology. 2018;5173-8. https://doi.org/10.1016/j.virol.2017.12.015

8. Alfaraj SH, Al-Tawfiq JA, Memish ZA. Middle East Respiratory Syndrome Coronavirus (MERS-CoV) infection during pregnancy: Report of two cases \& review of the literature. J Microbiol Immunol Infect. 2019;52(3):501-503. https://doi.org/10.1016/j.jmii.2018.04.005

9. Payne DC, Iblan I, Alqasrawi S et al. Stillbirth during infection with Middle East respiratory syndrome coronavirus. J Infect Dis. 2014;209(12):1870-1872. https://doi.org/10.1093/infdis/jiu068

10. Huang X, Wei F, Hu L et al. Epidemiology and Clinical Characteristics of COVID-19. Arch Iran Med. 2020;23(4):268-271. https://doi.org/10.34172/aim.2020.09

11. Guo YR, Cao QD, Hong ZS et al. The origin, transmission and clinical therapies on coronavirus disease 2019 (COVID-19) outbreak - an update on the status. Mil Med Res. 2020;7(1):11. https://doi.org/10.1186/s40779-020$\underline{00240-0}$

12. Zhou P, Yang XL, Wang XG et al. A pneumonia outbreak associated with a new coronavirus of probable bat origin. Nature. 2020;579(7798):270-273. https://doi.org/10.1038/s41586-020-2012-7 
13. di MG, Cristina S, Concetta R et al. SARS-Cov-2 infection: Response of human immune system and possible implications for the rapid test and treatment. Int Immunopharmacol. 2020;84:106519.

https://doi.org/10.1016/j.intimp.2020.106519

14. Shahid Z, Kalayanamitra R, McClafferty B et al. COVID-19 and Older Adults: What We Know. J Am Geriatr Soc. 2020;68(5):926-929. https://doi.org/10.1111/jgs.16472

15. Liu D, Li L, Wu X et al. Pregnancy and Perinatal Outcomes of Women With Coronavirus Disease (COVID-19) Pneumonia: A Preliminary Analysis. AJR Am J Roentgenol. 2020;215(1):127-132. https://doi.org/10.2214/AJR.20.23072

16. Pedersen JH, Saghir Z, Wille MM et al. Ground-Glass Opacity Lung Nodules in the Era of Lung Cancer CT Screening: Radiology, Pathology, and Clinical Management. Oncology (Williston Park). 2016;30(3):266-274.

17. Ferrazzi E, Frigerio L, Savasi V et al. Vaginal delivery in SARS-CoV-2-infected pregnant women in Northern Italy: a retrospective analysis. BJOG. 2020. https:// $\underline{\text { doi.org/10.1111/1471-0528.16278 }}$

18. Chen H, Guo J, Wang $\mathrm{C}$ et al. Clinical characteristics and intrauterine vertical transmission potential of COVID-19 infection in nine pregnant women: a retrospective review of medical records. Lancet. 2020;395(10226):809815. https://doi.org/10.1016/S0140-6736(20)30360-3

19. Khan S, Jun L, Nawsherwan et al. Association of COVID-19 with pregnancy outcomes in health-care workers and general women. [letter]. Clin Microbiol Infect. 2020;26(6):788-790. http://doi.org/10.1016/j.cmi.2020.03.034

20. Yu N, Li W, Kang Q et al. Clinical features and obstetric and neonatal outcomes of pregnant patients with COVID-19 in Wuhan, China: a retrospective, single-centre, descriptive study. Lancet Infect Dis. 2020;20(5):559-564. https://doi.org/10.1016/S1473-3099(20)30176-6

21. Breslin N, Baptiste C, Miller R et al. Coronavirus disease 2019 in pregnancy: early lessons. Am J Obstet Gynecol MFM. 2020;2(2):100111. https://doi.org/10.1016/j.ajogmf.2020.100111

22. Alzamora MC, Paredes T, Caceres D et al. Severe COVID-19 during Pregnancy and Possible Vertical Transmission. Am J Perinatol. 2020;37(8):861-865. https://doi.org/10.1055/s-0040-1710050

23. Zhu H, Wang L, Fang $\mathrm{C}$ et al. Clinical analysis of 10 neonates born to mothers with 2019-nCoV pneumonia. Transl Pediatr. 2020;9(1):51-60. https://doi.org/10.21037/tp.2020.02.06

24. Blencowe H, Cousens S, Oestergaard MZ et al. National, regional, and worldwide estimates of preterm birth rates in the year 2010 with time trends since 1990 for selected countries: a systematic analysis and implications. Lancet. 2012;379(9832):2162-2172. https://doi.org/10.1016/S0140-6736(12)60820-4

25. Qiancheng X, Jian S, Lingling P et al. Coronavirus disease 2019 in pregnancy. Int J Infect Dis. 2020;95376383. https://doi.org/10.1016/j.ijid.2020.04.065

26. Liu H, Liu F, Li J et al. Clinical and CT imaging features of the COVID-19 pneumonia: Focus on pregnant women and children. J Infect. 2020;80(5):e7-e13. https://doi.org/10.1016/j.jinf.2020.03.007

27. Wu C, Yang W, Wu X et al. Clinical Manifestation and Laboratory Characteristics of SARS-CoV-2 Infection in Pregnant Women. Virol Sin. 2020;35(3):305-310. https://doi.org/10.1007/s12250-020-00227-0

28. Liu H, Wang LL, Zhao SJ et al. Why are pregnant women susceptible to COVID-19? An immunological viewpoint. J Reprod Immunol. 2020;139:103122. https://doi.org/10.1016/j.jri.2020.103122

29. Schwartz DA. An Analysis of 38 Pregnant Women with COVID-19, Their Newborn Infants, and MaternalFetal Transmission of SARS-CoV-2: Maternal Coronavirus Infections and Pregnancy Outcomes. Arch Pathol Lab Med. 2020 https://doi.org/10.5858/arpa.2020-0901-SA

30. Panahi L, Amiri M, Pouy S. Risks of Novel Coronavirus Disease (COVID-19) in Pregnancy; a Narrative Review. Arch Acad Emerg Med. 2020;8(1):e34.

31. Di Mascio D, Khalil A, Saccone G et al. Outcome of Coronavirus spectrum infections (SARS, MERS, COVID 1 -19) during pregnancy: a systematic review and meta-analysis. Am J Obstet Gynecol MFM. 2020;2(2):100-107. https://doi.org/10.1016/j.ajogmf.2020.100107 